\title{
A Comparative Study of Piperacillin-Tazobactam With and Without Vancomycin as Empirical Therapy for Febrile Neutropenic Patients With Solid Tumor Malignancies
}

\author{
Mansoor Sirkhazi ${ }^{\mathrm{a}, \mathrm{b}, \mathrm{f}}$, Azmi Sarriff ${ }^{\mathrm{b}}$, Noorizan Abd Aziz ${ }^{\mathrm{c}}$, Fatma Almana ${ }^{\mathrm{a}}$, Osama Arafat ${ }^{\mathrm{a}}$, \\ Mahmoud Shorman ${ }^{\text {d, e }}$
}

\begin{abstract}
Background: The objective of this study was to evaluate the outcomes, mortality and toxicity associated with piperacillin-tazobactam (PT) and the addition of vancomycin (VM) to the empirical treatment of febrile neutropenic cancer patients.
\end{abstract}

Method: A retrospective study on adult febrile neutropenic patients who were admitted between September 2008 and May 2013 with solid tumor malignancies was conducted at King Fahad Specialist Hospital Dammam, Saudi Arabia.

Results: Out of 86 febrile neutropenic patients, 60 patients were treated with PT group and 26 patients were with PT + VM group. The two groups were comparable in terms of outcome, mortality, nephrotoxicities and hepatotoxicities. The median duration of neutropenia in PT treatment group was 4 days (range 1 - 10) in the female and 7 days (range $1-13$ ) in males while in PT + VM 6 days (range $1-5$ ) in female and 7.5 days (range $1-6$ ) in male with significance $\mathrm{P}=0.007$. There was no significant difference in terms of duration of fever and length of stay between the two treatment groups. There were no deaths reported during treatment in both groups. In PT, the microbial eradication was $27 / 40(67.5 \%)$ patients $(14 / 27(51.9 \%)$ of female and $13 / 27(48.1 \%)$ of male)), whereas it was $13 / 40(32.5 \%)$ patients $(9 / 13(69.2 \%)$ of female and $4 / 13(30.8 \%)$ of male)) in PT + VM group. Overall, there was no significant difference in terms of microbiological eradication between the two groups (OR: 1.22; $95 \%$ CI: $0.486-3.072 ; \mathrm{X}^{2}$ stat: $\left.0.182 ; \mathrm{P}=0.67\right)$. Response to therapy in clinically defined infections was higher 16/23 (69.56\%) in PT treat-

Manuscript accepted for publication December 11, 2014

aDepartment of Pharmacy, King Fahad Specialist Hospital, Dammam, Saudi Arabia

bSchool of Pharmaceutical Sciences, University Sains Malaysia, 11800 Minden, Penang, Malaysia

'Faculty of Pharmacy, University Technologia MARA, Selangor DE, Malaysia dInfection Control Department, King Fahad Specialist Hospital, Dammam, Saudi Arabia

eInternal Medicine Department, Marshall University, Huntington, WV, USA ${ }^{f}$ Corresponding Author: Mansoor Sirkhazi, Department of Pharmacy, King Fahad Specialist Hospital, Dammam, Saudi Arabia.

Email: mansoor_apc@yahoo.co.uk

doi: http://dx.doi.org/10.14740/wjon867w ment group than $7 / 23$ (30.44\%) in PT + VM group. But there was no significant difference between the two treatment groups in terms of clinically defined infections (OR: 1.013; 95\% CI: 0.359 - 2.862; X stat: $0.001 ; \mathrm{P}=0.98$ ). There was no significant difference in renal and liver functions between the two groups in terms of serum creatinine level and clearance, alkaline phosphate and alanine tranferase and gama glutamyl tranferase. The most commonly isolated organisms were Escherichais coli (eighteen isolates), Staphylococcus aureus (seven isolates), Streptococcus spp (six isolates) and Klebsiella pneumonia (four isolates). The overall success rate was similar in both treatment arms and treatment was well tolerated, with no severe adverse reactions reported.

Conclusion: Although the addition of VM might provide an additional value for coverage of gram-positive pathogens. This study demonstrates that there was no significant difference in terms of response rate in both treatment groups, which could be due to the low local methicillin-resistant Staphylococcus aureus (MRSA) rates and other resistant gram-positive organisms at our institution, stressing the importance of local antibiograms in developing empirical neutropenic fever protocols.

Keywords: Febrile neutropenia; Toxicities; Hematological malignancies; Piperacillin-tazobactam; Vancomycin

\section{Introduction}

Despite extensive clinical studies, none had recommended a single empirical therapy for the initial treatment of febrile neutropenic patients. The information from previous studies are outdated due to the tremendous changes in the bacterial etiology, sensitivity and resistance patterns as well as the criteria used to assess the treatment outcome. The effectiveness of antibiotic therapy may be influenced by the local patterns of bacterial infections and susceptibility $[1,2]$. By tradition, the broad-spectrum antimicrobial agents were used either as mono or combination therapy for empirical treatment in febrile neutropenic cancer patients. Many studies showed 50$70 \%$ of response rates with the empirical therapy [3]. It has been suggested that an appropriate empirical administration of broad-spectrum antibiotics might decrease the mortality rate caused by bacterial infections in febrile neutropenia [1]. In this 
regard, empiric monotherapy of an antipseudomonal $\beta$-lactam agent, such as piperacillin-tazobactam (PT), carbapenems or an anti-pseudomonal cephalosporins was recommended as initial management of febrile neutropenia, particularly for the high-risk patients. The addition of vancomycin (VM) to betalactam antibiotics as initial therapy is recommended for the management of infectious complications (e.g. hypotension and pneumonia) or if gram-positive microorganism resistance is suspected or proven. Glycopeptide antibiotics are recommended for specific conditions, including suspected catheter-related infection, skin and soft tissue infection, pneumonia, or hemodynamic instability and where the gram-positive organisms are predominant. Despite of these advances in the management of febrile neutropenia empirical treatment still remains challenging due to changes in bacterial etiology [4-7]. Despite these advances in the management of febrile neutropenia, it still carries a high morbidity and mortality [8].

In our literature review, we found that most of the studies from different parts of the world evaluated the effectiveness and safety of PT with or without aminoglycosides [9-16]. Fewer studies analyzed glycopeptides plus PT combination as initial empirical therapy for treatment of febrile neutropenic episodes in febrile neutropenic cancer patients [1, 17-20]. Furthermore, the addition of VM to $\beta$-lactam antibiotic therapy would add toxicities and emergence of VM-resistant enterococci $[1,21]$.

In this study, we analyzed the outcome variables and the difference in rate of response between PT with and without the addition of VM.

\section{Patients and Methods}

\section{Study design}

Retrospective study on adult febrile neutropenic patients with solid tumor malignancies, who were treated with PT and PT plus VM at King Fahad Specialist Hospital, Dammam Saudi Arabia.

\section{Inclusion criteria}

Eligible patients were those with fever defined as the elevation of single oral temperature measurement of equal or greater than $38.3^{\circ} \mathrm{C}\left(101^{\circ} \mathrm{F}\right)$ or a temperature of equal or greater than $38.0^{\circ} \mathrm{C}\left(100.4^{\circ} \mathrm{F}\right)$ sustained over a 1 -h period. Neutropenia was defined as absolute neutrophil count (ANC) of less than 500 cells $/ \mathrm{mm}^{3}$ or predicted decrease below 500 cells $/ \mathrm{mm}^{3}$ during the next $48 \mathrm{~h}$. Male and female over the age of 18 years with solid tumor malignancies were included. Patients with presumed infectious cause of fever were included as high risk.

\section{Exclusion criteria}

Patients who have no evidence of neutropenia, history of aller- gic reactions to any of antimicrobial in the study, and patients who received any other antimicrobials and investigational drugs within $72 \mathrm{~h}$ were excluded. The study was approved by the hospital Institutional Reweive Board (IRB) committee. All the data were collected from electronic hospital information system, MedicaPlus and confirmed with review of the medical chart. All microbiology reports were based on Clinical and Laboratory Standard Institute (CLSI) guidelines.

\section{Classification of febrile episodes}

Febrile episodes were categorized into three groups: 1) microbiologically defined infections (MDI): fever with microbial isolation; 2) clinically defined infections (CDI): defined site of infection (pneumonia, enterocolities or cellulite) but no microbiological confirmation and 3) fever of unknown origin (FUO): patients have a fever with no clinical evidence, nor microbial documented infection.

\section{Evaluation of response}

The primary outcome was treatment success without modification or addition of other antibiotics to the initial treatment regimen within $72 \mathrm{~h}$ of therapy. Success was assessed after 72 $h$ of antibacterial therapy.

\section{Effectiveness of therapy}

Effectiveness of therapy was defined as complete resolution of fever (reduction in temperature $<38{ }^{\circ} \mathrm{C}$ when measured orally and sustained for $48 \mathrm{~h}$ ), clinical signs and symptoms of infection and eradication of any infectious organisms, without changes in initial assigned therapy. The bacteriological response was assessed after $72 \mathrm{~h}$ of treatment and overall response was assessed 7 days after the end of therapy.

\section{Failure of therapy}

The treatment was considered as a failure if the antimicrobial therapy was modified by the addition of other antibiotics or discontinuation of initial empirical therapy and continuation with other antibiotics during the $72 \mathrm{~h}$ of treatment. The failure of therapy is also defined as persistent fever in a patient with signs of clinical deterioration, microbiological evidence, and clinical progression of the presumed infection or adverse event associated with the antibiotic regimen. Death occurring within $72 \mathrm{~h}$ of treatment is considered as failure of both clinical and bacterial response.

\section{Toxicities}

The safety of therapy was evaluated by monitoring laboratory values. Nephrotoxicity and hepatotoxicity are defined as an in- 


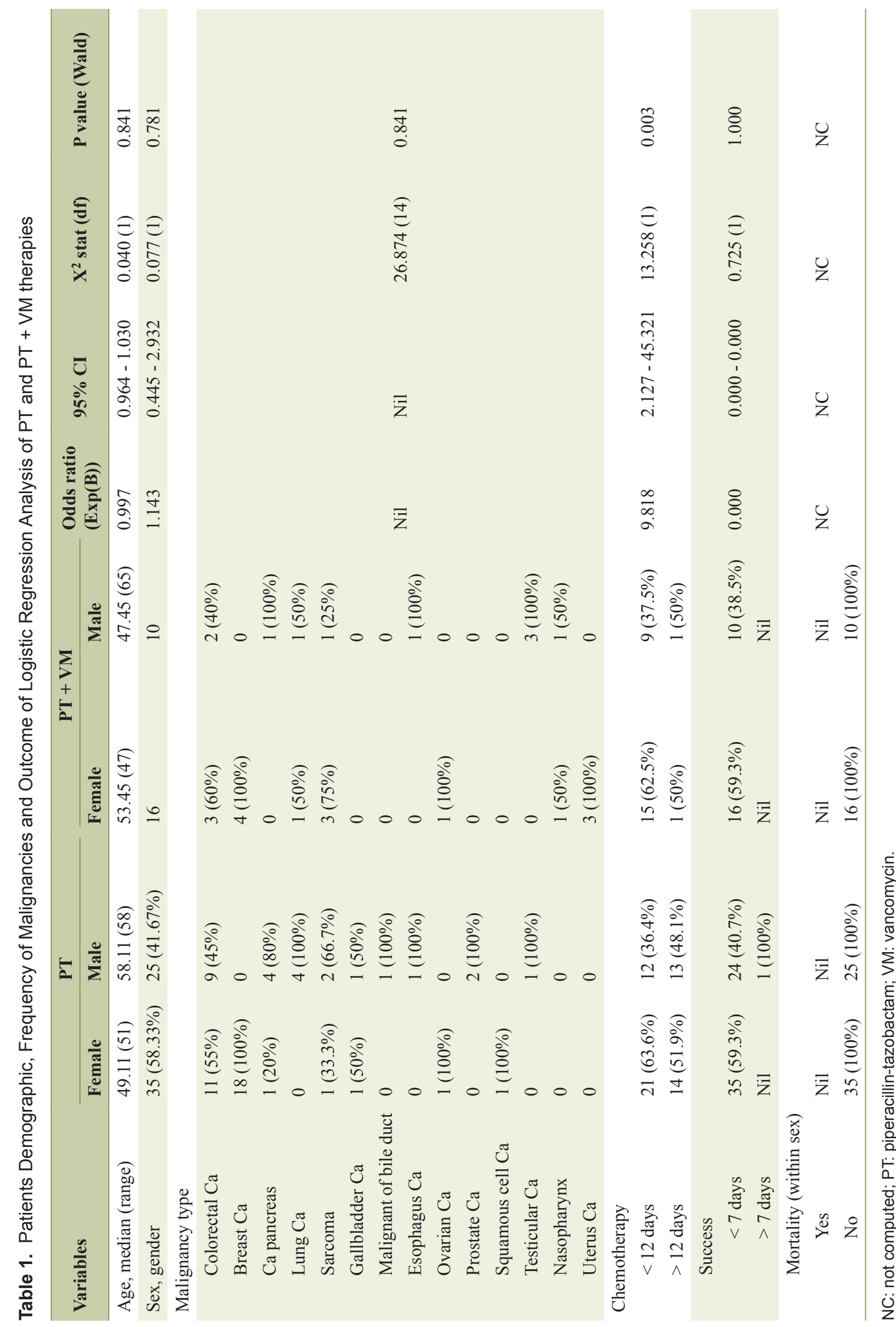


Table 2. Response to Therapy of PT Versus PT + VM Treatment Groups

\begin{tabular}{|c|c|c|c|c|c|c|c|c|c|}
\hline \multirow{2}{*}{$\begin{array}{l}\text { Effectiveness } \\
\text { variables }\end{array}$} & \multirow{2}{*}{ Baseline } & \multicolumn{2}{|c|}{ PT } & \multicolumn{2}{|c|}{$\mathbf{P T}+\mathrm{VM}$} & \multirow{2}{*}{$\begin{array}{l}\text { Odds } \\
\text { ratio }\end{array}$} & \multirow{2}{*}{$95 \%$ CI } & \multirow{2}{*}{$\begin{array}{l}X^{2} \text { stat } \\
\text { (df) }\end{array}$} & \multirow{2}{*}{ P value } \\
\hline & & Frequency $(\mathbf{F})$ & Frequency (M) & Frequency $(\mathrm{F})$ & Frequency (M) & & & & \\
\hline \multirow[t]{2}{*}{ Fever } & $<38.1{ }^{\circ} \mathrm{C}$ & $35(58.3 \%)$ & $25(41.7 \%)$ & $16(61.5 \%)$ & $10(38.5 \%)$ & $\mathrm{NC}$ & $\mathrm{NC}$ & $\mathrm{NC}$ & $\mathrm{NC}$ \\
\hline & $>38.1^{\circ} \mathrm{C}$ & Nil & Nil & Nil & Nil & & & & \\
\hline \multirow{2}{*}{$\begin{array}{l}\text { Pleural ef- } \\
\text { fusion }\end{array}$} & Yes & $9(56.2 \%)$ & $7(43.8 \%)$ & $3(50 \%)$ & $3(50 \%)$ & 0.825 & $0.281-2.422$ & $0.124(1)$ & 0.726 \\
\hline & No & $26(59.1 \%)$ & $18(40.9 \%)$ & $13(65 \%)$ & $7(35 \%)$ & & & & \\
\hline Consolidation & Yes & $5(14.3 \%)$ & $4(16 \%)$ & $4(25 \%)$ & $3(30 \%)$ & 2.088 & $0.682-6.395$ & $1.621(1)$ & 0.197 \\
\hline
\end{tabular}

crease in serum creatinine, transaminases, bilirubin or alkaline phosphatase by at least twice the upper normal limit [14, 22]. Hypokalemia was defined as an increase in serum potassium level $>10 \mathrm{mmol}$ below the lower limit of the normal range [23].

\section{Statistical analysis}

The primary analysis was a comparison of response rate for PT and PT + VM treatment groups. Data were analyzed by using descriptive statistical methods. Categorical variables were expressed as frequencies and percentages. The two groups were compared with Student's $t$ test for normally distributed data and not normally distributed data by Krsukal-Wallis $\mathrm{H}$ rank sum tests (non-parametric tests). The continuous variable values that normally and not normally distributed were expressed as median and range. Univariate logistic binary regression was used to determine the predictor variables associated with the effectiveness and safety of antibiotics in infections with febrile neutropenic cancer patients from the set of predictor variables. The significance between two groups was studied by using Wald $\mathrm{X}^{2}$ stat for categorical data whenever appropriate. The statistical significant variables in univariate analysis were included in the multivariate logistic regression analysis as stepwise selection was performed at a significant level of $\mathrm{P}<0.2$, to avoid any missing variables, which have strong relationship for effectiveness and safety, to determine the predictive variable for effectiveness and safety. In all analysis, an alpha level of $\mathrm{P}<0.05$ indicated statistical significance with $95 \% \mathrm{CI}$, whenever appropriate, calculated for the assessment of differences in response rates between PT versus PT $+\mathrm{VM}$ in terms of outcome and mortality. If $\mathrm{P} \leq 0.05$, there is a significant difference in response rate between the variables of two treatment groups and if $\mathrm{P}>0.05$, indicates that there is a no significant difference of outcome variables between two arms. Statistical analysis was performed by using SPSS software, version 20.0 package (statistical program for social science, version 20.0, Chicago, IL, USA).

\section{Results}

\section{Characteristics of patients and type of cancers}

From September 2008 to May 2013, the total of 95 febrile neu- tropenic patients were evaluated, and 86 febrile neutropenic patients were eligible for the study. Nine patients were excluded from the study because of modification in initial empirical therapy and protocol violation, five patients received levofloxacin, and two patients had added imipenem-cilastatin, one patient had linezolid and another patient was on meropenem plus VM which were started by the primary physician. Overall 86 febrile neutropenic patients were assessed for response to therapy, of which $60 / 86(69.77 \%)$ of patients received PT and 26/86 (30.23\%) were treated with PT plus VM. The two groups were assessed for the response to antibacterial therapy in terms of age, sex, clinical outcome, mortality and overall success. The median age of female and male was 49.11 and 58.11 years in PT group, whereas it was 53.45 and 47.45 in the $\mathrm{PT}+\mathrm{VM}$ group $(\mathrm{P}=0.841)$.

The most common underlying malignancies were colorectal, breast and lung cancers. There were no significant differences between the two treatment groups with respect to age, sex and underlying malignancies. Fifty-seven patients develop neutropenia within less than 12 days of chemotherapy. Chemotherapy-induced (CI) neutropenic patients were higher in PT treatment groups (33 patients) than in a PT + VM treatment group. Females had higher risk of developing CI neutropenia than male. Patient's response to therapy was higher in the PT group compared to the PT + VM group (OR: 9.818; 95\% CI: $\left.2.12-45.32 ; \mathrm{X}^{2}: 13.25 ; \mathrm{P}=0.003\right)$. The details are illustrated in Table 1.

\section{Clinical response, site of infection and microbial distribu- tion}

The distribution of infection categories did not differ considerably in the two treatment groups. Clinically documented infection occurred in 16 patients, nine $(56.2 \%)$ females and seven $(43.8 \%)$ males in PT group and in seven patients, four $(57.1 \%)$ females, three $(42.9 \%)$ males in $\mathrm{PT}+\mathrm{VM}(\mathrm{P}=0.98)$ groups, respectively. No sign of the fever was found in 24 patients, $15(62.5 \%)$ females, nine $(37.5 \%)$ males in PT group and 10 patients, five $(50 \%)$ females, five $(50 \%)$ males in PT + VM groups $(\mathrm{P}=0.89)$, respectively. The pleural effusion and Lung consolidation were found in $9 / 16$ patients in $\mathrm{PT}$ group $(\mathrm{P}=$ $0.72)$ and $6 / 7$ patients in $\mathrm{PT}+\mathrm{VM}$ group $(\mathrm{P}=0.19)$. There is no significant differences between two groups with respect to response to therapy (Table 2).

The duration of fever was longer for PT + VM group with a median of 2 days, range 1 - 5 days as compared to a median 


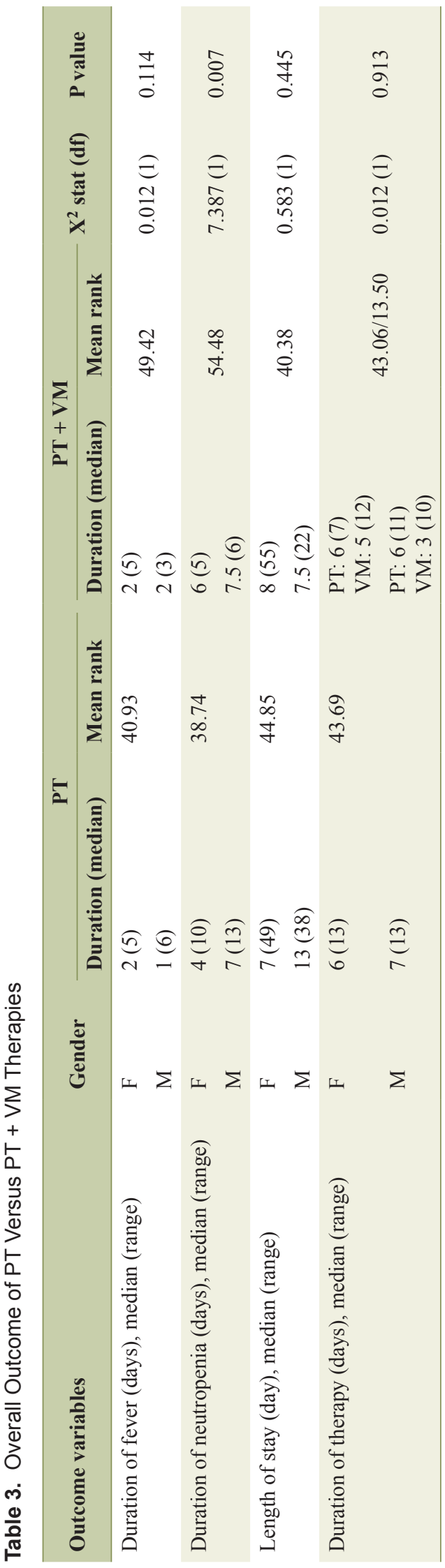

of 1 day, range 1 - 6 days in PT group. The duration of fever was slightly higher in females. However, it was noted that there was no statistical differences between the two groups $(\mathrm{P}=$ $0.114)$. Although there was no difference in the length of stay, but it was considerably higher in males, with a median of 13 days, range 1 - 38 days in the PT group $(\mathrm{P}=0.44)$. Also it was noted that there was no significant differences in the duration of therapy between the two groups $(\mathrm{P}=0.91)$. The duration of neutropenica was found to be higher in the female's with a median of 6 days, range $1-5$ days, with mean rank 54.48 in the PT + VM group compared to PT group with median, 4 days, range 1 - 10 days, mean rank $38.74(\mathrm{P}=0.007)$ (Table 3$)$.

Out of 40 febrile neutropenic patients with microbiologically documented infections, 30 gram-negative pathogens were isolated from $27 / 60(45 \%)$ of patients $(14 / 27(51.9 \%)$ were female and 13/27 (48.1\%) were male) and 20 gram-positive microorganisms were isolated from $13 / 26(50 \%)$ of patients $(9 / 26(69.2 \%)$ female and $4 / 26(30.8 \%)$ male). The $60 \%$ of gram-negative isolates and $40 \%$ of gram-positive isolates were treated with PT group, whereas $60 \%$ of gram-positive and $40 \%$ of gram-negative infections were treated with PT + VM group. The types of organisms causing infection in the two treatment arms were similar, with slightly more gram-negative bacteremia. There was no statistical significance with respect to microbial eradication between the two treatment arms (OR: 1.22; $95 \%$ CI: 0.486 - 3.072; $\left.\mathrm{X}^{2}: 0.18 ; \mathrm{P}=0.67\right)$.

Out of 30 gram-negative isolates, the common pathogens were Escherichia coli $(\mathrm{n}=18)$, Klebsiella pneumonia $(\mathrm{n}=4)$ and Pseudomonas spp $(\mathrm{n}=3)$. The susceptibility to PT was $79.92 \%, 83.23 \%$ and $69.23 \%$ for E. coli, Pseudomonas spp and Klebsiella pneumonia respectively. Gram-positive pathogen susceptibility to VM was $100 \%$ for Staphylococcus aureus and Streptococcus spp, Staphylococcus spp $99.8 \%$ and Enterococcus spp $98.85 \%$. The details of susceptibility and resistance patterns are listed in Table 4. The most common isolation sites were bloodstream followed by urine, wound, body fluids and sputum.

Overall, the improvement rate with or without VM in initial empirical therapy was 59/60 (98.33\%) in PT and $100 \%$ in PT + VM. There is no drug-induced mortality during treatment.

There was no major difference in electrolyte imbalance, namely, magnesium and potassium. Blood urea nitrogen was elevated in PT group, but multivariate logistic regression shows that there was no significant difference between PT and $\mathrm{PT}+\mathrm{VM}$ treatment groups $(\mathrm{P}=0.114)$.

In this study, we developed two models, model I, which is the relationship between outcome and variables (univariate logistic regression) and model II, for determining the predictor variables for a specific outcome (multivariate logistic regression) as shown in Table 5.

\section{Model I: univariate logistic regression for predictor vari- able}

Univariate logistic regression analysis identified 10 variables with $P$ value of less than 0.2 , which includes patients on chemotherapy, consolidation in lung, blood pressure, white blood 
Table 4. Bacterial Spectrum, Susceptibility and Resistance Patterns of Microorganisms

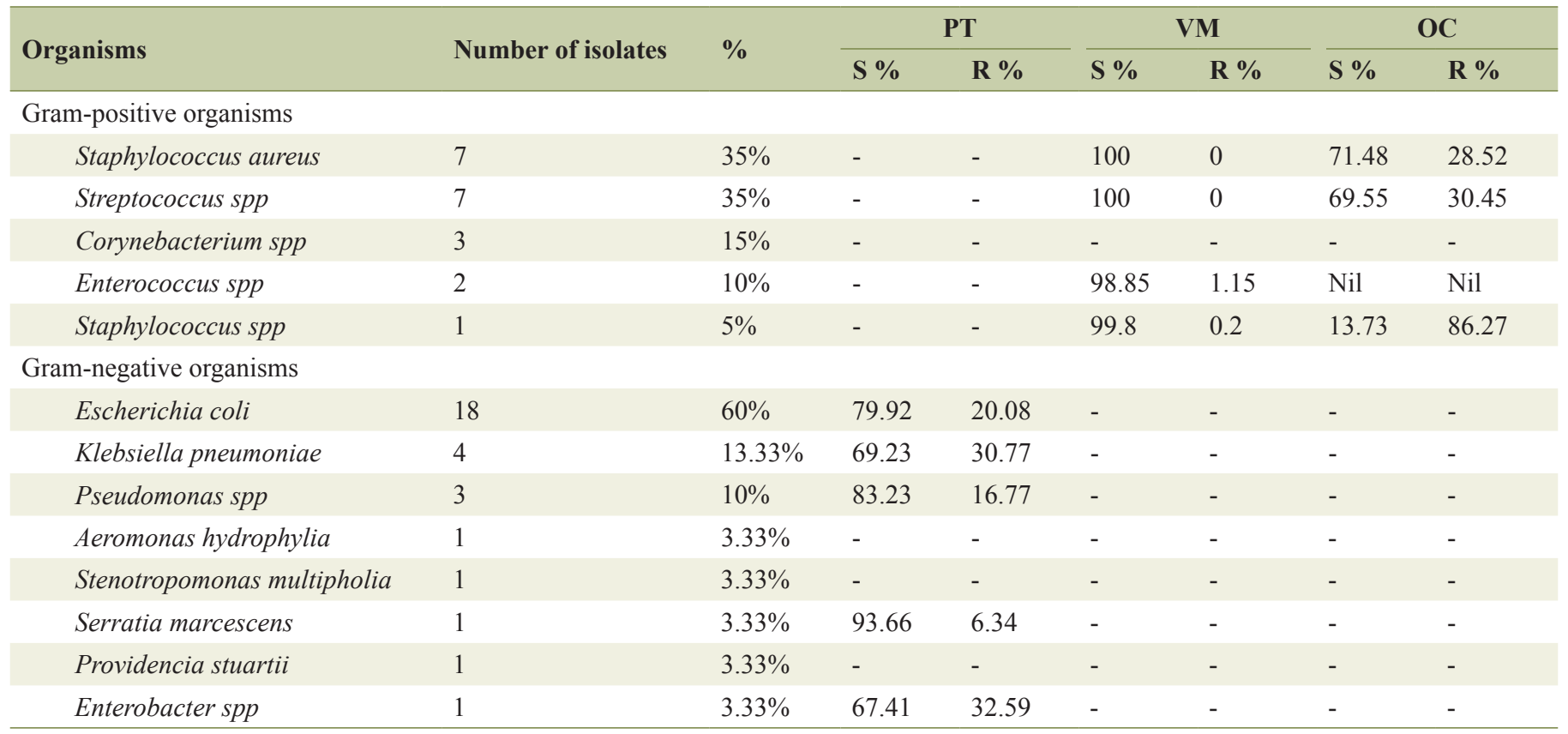

cells (WBC), hemoglobin, blood urea nitrogen, serum creatinine level, alkaline phosphatase, aspartate aminotransferase and gama glutamyl transpeptidase. These variables were further analyzed with the multivariate logistic regression model to determine the dependent predictor variables for effectiveness of study antibiotics. Univariate logistic regression revealed that the response to PT therapy was almost OR of 9.818 more likely among subjects who develop neutropenia less than 12 days of chemotherapy regimen $(\mathrm{P}=0.003)(95 \% \mathrm{CI}: 2.12$
- 45.32). Similarly, the success was almost OR of 2.088 more likely among patients who develop Lung consolidation with no significant difference $(\mathrm{P}=0.197)(95 \% \mathrm{CI}$ : 0.682 - 6.395), as shown in Table 5.

The elevated aspartate aminotransferase was nearly OR of 6.25 with $95 \%$ CI of 0.768 - 50.860 (0.087), increase in alkaline phosphatase was closely OR of 5.111 with $95 \%$ CI of 1.380 - $18.929(0.015)$ and the increase in gama glutamyl transpeptidase level was almost OR of 2.539 with $95 \%$ CI of

Table 5. Logistic Regression Analysis of Variables Associated With Treatment Success Without Treatment Modification

\begin{tabular}{|c|c|c|c|c|}
\hline Variables & $\begin{array}{l}\text { Univariate OR } \\
(95 \% \mathrm{CI})\end{array}$ & $\begin{array}{l}\text { Univariate } \\
\text { (P) }\end{array}$ & $\begin{array}{l}\text { Multivariate OR } \\
(95 \% \mathrm{CI})\end{array}$ & $\begin{array}{l}\text { Multivariate } \\
\text { (P) }\end{array}$ \\
\hline Chemotherapy, $<12$ days vs. $>12$ days & $9.818(2.127-45.32)$ & 0.003 & $3.442(1.29-754.09)$ & 0.034 \\
\hline $\begin{array}{l}\text { Blood pressure, baseline: } 90 / 60 \text { to } 130 / 90 \\
\mathrm{~mm} \mathrm{Hg} \text { vs. }<80 / 50 \text { and }>200 / 120 \mathrm{~mm} \mathrm{Hg}\end{array}$ & $0.078(0.024-0.258)$ & 0.001 & $-3.094(0.005-0.386)$ & 0.005 \\
\hline $\begin{array}{l}\text { WBC, baseline: } 4.5-11 \times 10^{9} / \mathrm{L} \\
\text { vs. }<4.5-11 \times 10^{9} / \mathrm{L}\end{array}$ & $0.233(0.084-0.652)$ & 0.005 & $-1.923(0.021-1.01)$ & 0.051 \\
\hline $\begin{array}{l}\text { Hemoglobin, baseline: } 13.5-17.5 \mathrm{~g} / \\
\text { dL vs. }<13.5-17.5 \mathrm{~g} / \mathrm{dL}\end{array}$ & $0.146(0.053-0.404)$ & 0.001 & $-2.628(0.011-0.477)$ & 0.006 \\
\hline $\begin{array}{l}\text { Blood urea nitrogen, baseline: } 2.7-7.2 \\
\text { mmol vs. }<2.7->7.2 \mathrm{mmol}\end{array}$ & $0.267(0.102-0.700)$ & 0.007 & $-1.343(0.049-1.382)$ & 0.114 \\
\hline $\begin{array}{l}\text { Serum creatinine level, baseline: F, } 47-115 / \mathrm{M} \text {, } \\
53-88 \mathrm{mmol} / \mathrm{L} \text { vs. }>47-115 / \mathrm{M},>53-88 \mathrm{mmol}\end{array}$ & $3.319(0.692-15.919)$ & 0.134 & $-0.173(0.046-15.466)$ & 0.907 \\
\hline GGT, baseline: 15 - $85 \mathrm{U} / \mathrm{L}$ vs. $>85 \mathrm{U} / \mathrm{L}$ & $2.539(0.931-6.928)$ & 0.069 & $0.422(0.14-16.174)$ & 0.726 \\
\hline
\end{tabular}

ALP: alkaline phasphatase; AST: aspartate aminotransferase; GGT: gama-glutamyl transepiptidase. 
$0.931-6.928(\mathrm{P}=0.069)$, occurring in patients treated with PT therapy. There was considerable difference in elevation of alkaline phosphatase in patients who treated with PT therapy. The increase in serum creatinine was almost OR of 3.319 with $95 \%$ CI of $0.692-15.919$ but there is no significant difference between the two treatment groups $(\mathrm{P}=0.134)$ as shown in Table 5.

\section{Model II: multivariate logistic regression for predictor variables}

The multivariate logistic regression analysis was performed to estimate the predictor variables for effectiveness and treatment success of studied antibiotics. Among tested predictor variables in univariate analysis, the five variables were statistically significant predictors of effectiveness: blood pressure $(\mathrm{P}$ $=0.005)$, hemoglobin $(P=0.006)$, consolidation $(P=0.026)$, patients on chemotherapy $(\mathrm{P}=0.034)$ and $\mathrm{WBC}(\mathrm{P}=0.051)$ as shown in Table 5.

Of these variables, the patients who develop neutropenia within 12 days of chemotherapy administration were improved with OR of 3.442 with $95 \%$ CI of $1.29-754.09(\mathrm{P}=0.034)$ in PT group. The odd of treatment success with PT was OR 2.305 with $95 \%$ CI of 1.323 - 75.003 in patients who develop consolidation $(\mathrm{P}=0.026)$. The success rate was higher in $\mathrm{PT}$ than $\mathrm{PT}+\mathrm{VM}$ group, which is statistically significant.

\section{Toxicity}

With regard to toxicity, the odds increase in alkaline phosphatase level with PT therapy was nearly 2.363 with $95 \% \mathrm{CI}$ of $0.571-193.476(\mathrm{P}=0.113)$ and with $\mathrm{PT}+\mathrm{VM}$ therapy was 1.546 with $95 \%$ CI of $0.051-432.207(\mathrm{P}=0.503)$, which is statistically not significant. The comparison of univariate and multivariate logistic regression outcome variables of PT vs. PT + VM treatment groups was shown in the Table 5. The increase in blood urea nitrogen was almost similar in both treatment arms which was statistically significant $(\mathrm{P}=0.007)$. The multivariate logistic regression analysis shows no statistical significant with $95 \% \mathrm{CI}$ of $0.049-1.382(\mathrm{P}=0.114)$ in subjects treated with PT and PT + VM groups as shown in Table 5.

\section{Discussion}

Over the past 40 years, the standard treatment regimen in febrile neutropenia has been changed in response to the emergence of new organisms [24]. The concept of combination therapy has been widely accepted because of their synergistic action and broad coverage of gram-positive and negative organisms [8, 25]. Many investigators support the theory of synergism of antibiotic combinations [26]. Most commonly used combination therapies, namely, an anti-pseudomonal beta lactam (PT, cefepime, ceftazidime and carbapenems) plus an aminoglycosides (amikacin, gentamycin or tobramycin) is one of the most recommended regimens in the treatment of high risk febrile neutropenic cancer patients [2, 25]. A major drawback of combination therapy is the increased likelihood of toxicities and associated costs $[8,25]$. The addition of glycopeptides, VM, is not recommended as a routine part of initial therapy because it may increase the risk of complications, namely, drug toxicities, fungal super infections and therefore it should be considered carefully $[5,12]$. Currently, the major issue is whether VM should be included as a part of initial therapy to cover for resistant gram-positive pathogens in the case of suspected catheter-related infections, skin and soft tissue infections and pneumonia or hemodynamic instability [5, $8,25,27]$. European Organization for Research and Treatment of Centers (EORTC) recommends that the addition of VM to initial therapy should be considered where gram-positive organisms are predominant $[5,12,27]$. In addition, the various studies suggested that addition of VM is probably not needed as a part of initial therapy or should be stopped unless there is gram-positive organisms from blood cultures. Most of grampositive organisms are not lethal $[20,27,28]$.

The main concern of our study was to evaluate the effectiveness of PT with or without VM as an initial therapy in terms of response to therapy, mortality and drug toxicities.

In this study, the resolution of fever was observed in 86 febrile episodes, and in PT group the median duration of fever was 1 day (range, 1 - 6) as compared with 2 days (range, 1 - 5) in the PT + VM group. The overall response was similar in both therapies. There is no significant difference between the two treatment arms in terms of resolution of fever $(\mathrm{P}=0.114)$. The finding in this study is consistent with previous studies [1, $11,20,26,29]$. The response to therapy is directly related to neutrophil count and trend [17]. The absolute neutrophil count was improved 100\% (60/60) in PT group and slightly lower $96.15 \%(25 / 26)$ in the PT + VM group $(\mathrm{P}=1.000)$. The duration of neutropenia was slightly higher in the PT + VM group than PT group. There is a significant difference in terms of median duration of neutropenia in the two treatment arms ( $P$ $=0.07)$. Many studies reported the duration of neutropenia for PT but fewer studies were discussed in PT + VM therapy [1, $14,17,24,30]$. The median length of stay was almost similar in two treatment groups. Though the length of stay was (median, 13 days) slightly higher in males with PT group but statistically there is no significant difference in two treatment arms $(\mathrm{P}=0.445)$. The result is almost similar with previous studies $[11,24]$.

PT is highly effective against gram-positive bacteria, including $\beta$ lactamase producing strains. It inhibits wide spectrum of gram-negative bacilli, including pseudomonas aerugenosa [9]. Del Favero et al 2001 reported the eradication of bacteremia due to $E$. coli was $42 \%$ in PT vs. $53 \%$ in imipenem-cilastatin. Mouton and associates performed an open, noncomparative study at 36 sites from six countries, with using PT, clinical cure occurred in $96 \%$ and bacterial eradication was noted in $93 \%$ of the cases. In the same study 15 pathogens were susceptible and all of them eradicated. In another study with PT, clinical cure rate was $74 \%$ and microbial eradication was 70\%. Harter et al 2005 reported in their study that there was no difference in eradication of gram-positive pathogens between PT versus ceftazidime. Several studies from different centers have shown that PT was effective against gram-posi- 
tive and negative pathogens $[9,17,26,29,31]$. VM should be included as a part of initial therapy in cancer institutions where gram-positive bacteria is a problem $[20,21]$. In one study, the response rate of VM was $66 \%$ and another study, $100 \%$ in severe gram-positive infections in febrile neutropenic cancer patients [19]. Cometta et al 2003 in their study concluded that the empirical addition of VM therapy is of benefit for grampositive bacterial infection that is resistant to PT; the glycopeptides had no effect on the time to defervescence, resolution of fever and all-cause mortality [1]. It is reasonable to include VM in empirical therapy where gram-positive organisms were predominant [1]. VM is a drug of choice with predictable activity against MRSA [25].

In our study, the predominance of gram-negative bacteria compared to resistant gram-positive bacteria explains the results of no added benefit of empirical VM. Overall in 86 febrile neutropenic patients, 30 isolates were gram-negative and 20 were gram-positive, and seven isolates of Staphylococcus aureus were methicillin-resistant (MRSA). The microbial eradication rate was 50\% (27/60) in PT + VM and 45\% (27/60) in PT group. Our study results were consistent with previous studies. To our knowledge, in our literature review, we did not find enough clinical studies, especially at local setting on comparison of PT versus PT + VM in febrile neutropenic patients with solid tumor malignancies.

In our study, we found no major difference in electrolyte imbalance, namely, magnesium and potassium. Blood urea nitrogen was elevated with the PT group, but multivariate regression showed no significant difference between PT and PT $+\mathrm{VM}$ groups $(\mathrm{P}=0.114)$. Some studies reported that the electrolyte imbalance is due to aggressive chemotherapy and the hypokelamia was found only in $4 \%$ of the population receiving PT therapy $[32,33]$.

The majority of studies was reported that the increase in serum creatinine level was found with the addition of VM [1, 20]. Some studies reported no difference in toxicities with the addition of VM $[1,17]$. The present study found that the overall success rates of therapy were similar in both the groups.

\section{Conclusion}

PT has broad spectrum of activity against gram-positive and gram-negative infections. The addition of VM should be considered where resistant gram-positive bacterial infection are encountered, based on the local epidemiology and antibiograms. The routine addition of VM may increase toxicities without changing the clinical outomes.

\section{References}

1. Cometta A, Kern WV, De Bock R, Paesmans M, Vandenbergh M, Crokaert F, Engelhard D, et al. Vancomycin versus placebo for treating persistent fever in patients with neutropenic cancer receiving piperacillin-tazobactam monotherapy. Clin Infect Dis. 2003;37(3):382-389.

2. Serefhanoglu K, Ersoy Y, Serefhanoglu S, Aydogdu I, Kuku I, Kaya E. Clinical experience with three combi- nation regimens for the treatment of high-risk febrile neutropenia. Ann Acad Med Singapore. 2006;35(1):1116.

3. Shamsi TS, Farzana T, Ansari SH, Ahmed A, Ishaque A. Febrile Neutropenia in Haematological Disorders: a single center review of Antibiotic policy and the Outcome. J Pak Med Assoc. 2003;53:190-193.

4. Eltahawy AT. Febrile neutropenia. Etiology of infection, empirical treatment and prophylaxis. Saudi Med J. 2003;24(4):331-336

5. Freifeld AG, Bow EJ, Sepkowitz KA, Boeckh MJ, Ito JI, Mullen CA, Raad, II, et al. Clinical practice guideline for the use of antimicrobial agents in neutropenic patients with cancer: 2010 update by the infectious diseases society of america. Clin Infect Dis. 2011;52(4):e56-93.

6. Ramphal R, Gucalp R, Rotstein C, Cimino M, Oblon D. Clinical experience with single agent and combination regimens in the management of infection in the febrile neutropenic patient. Am J Med. 1996;100(6A):83S-89S.

7. Roohullah A, Moniwa A, Wood C, Humble M, Balm M, Carter J, Weinkove R. Imipenem versus piperacillin/ tazobactam for empiric treatment of neutropenic fever in adults. Intern Med J. 2013;43(10):1151-1154.

8. Hathorn JW, Lyke K. Empirical treatment of febrile neutropenia: evolution of current therapeutic approaches. Clin Infect Dis. 1997;24(Suppl 2):S256-265.

9. Sanders WE, Jr., Sanders CC. Enterobacter spp.: pathogens poised to flourish at the turn of the century. Clin Microbiol Rev. 1997;10(2):220-241.

10. Frank U, Mutter J, Schmidt-Eisenlohr E, Daschner FD. Comparative in vitro activity of piperacillin, piperacillinsulbactam and piperacillin-tazobactam against nosocomial pathogens isolated from intensive care patients. Clin Microbiol Infect. 2003;9(11):1128-1132.

11. Bow EJ, Rotstein C, Noskin GA, Laverdiere M, Schwarer AP, Segal BH, Seymour JF, et al. A randomized, openlabel, multicenter comparative study of the efficacy and safety of piperacillin-tazobactam and cefepime for the empirical treatment of febrile neutropenic episodes in patients with hematologic malignancies. Clin Infect Dis. 2006;43(4):447-459.

12. Paul M, Yahav D, Fraser A, Leibovici L. Empirical antibiotic monotherapy for febrile neutropenia: systematic review and meta-analysis of randomized controlled trials. J Antimicrob Chemother. 2006;57(2):176-189.

13. Del Favero A, Menichetti F, Martino P, Bucaneve G, Micozzi A, Gentile G, Furno P, et al. A multicenter, doubleblind, placebo-controlled trial comparing piperacillintazobactam with and without amikacin as empiric therapy for febrile neutropenia. Clin Infect Dis. 2001;33(8):12951301.

14. Harter C, Schulze B, Goldschmidt H, Benner A, Geiss HK, Hoppe-Tichy T, Ho AD, et al. Piperacillin/tazobactam vs ceftazidime in the treatment of neutropenic fever in patients with acute leukemia or following autologous peripheral blood stem cell transplantation: a prospective randomized trial. Bone Marrow Transplant. 2006;37(4):373-379.

15. Sanz MA, Bermudez A, Rovira M, Besalduch J, Pascual 
MJ, Nocea G, Sanz-Rodriguez C. Imipenem/cilastatin versus piperacillin/tazobactam plus amikacin for empirical therapy in febrile neutropenic patients: results of the COSTINE study. Curr Med Res Opin. 2005;21(5):645655.

16. Leibovici L, Paul M, Poznanski O, Drucker M, Samra Z, Konigsberger H, Pitlik SD. Monotherapy versus betalactam-aminoglycoside combination treatment for gramnegative bacteremia: a prospective, observational study. Antimicrob Agents Chemother. 1997;41(5):1127-1133.

17. Jadeja L, Bolivar R, Fainstein V, Keating M, McCredie $\mathrm{K}$, Hay M, Bodey GP. Piperacillin plus vancomycin in the therapy of febrile episodes in cancer patients. Antimicrob Agents Chemother. 1984;26(3):295-299.

18. Rubin M, Hathorn JW, Marshall D, Gress J, Steinberg SM, Pizzo PA. Gram-positive infections and the use of vancomycin in 550 episodes of fever and neutropenia. Ann Intern Med. 1988;108(1):30-35.

19. Wood MJ. The comparative efficacy and safety of teicoplanin and vancomycin. J Antimicrob Chemother. 1996;37(2):209-222.

20. Feld R. Vancomycin as part of initial empirical antibiotic therapy for febrile neutropenia in patients with cancer: pros and cons. Clin Infect Dis. 1999;29(3):503-507.

21. Paul M, Dickstein Y, Borok S, Vidal L, Leibovici L. Empirical antibiotics targeting Gram-positive bacteria for the treatment of febrile neutropenic patients with cancer. Cochrane Database Syst Rev. 2014;1:CD003914.

22. Raad, II, Abi-Said D, Rolston KV, Karl CL, Bodey GP. How should imipenem-cilastatin be used in the treatment of fever and infection in neutropenic cancer patients? Cancer. 1998;82(12):2449-2458.

23. Ariffin H, Arasu A, Mahfuzah M, Ariffin WA, Chan LL, Lin HP. Single-daily ceftriaxone plus amikacin versus thrice-daily ceftazidime plus amikacin as empirical treatment of febrile neutropenia in children with cancer. J Paediatr Child Health. 2001;37(1):38-43.

24. Oztoprak N, Piskin N, Aydemir H, Celebi G, Akduman D, Keskin AS, Gokmen A, et al. Piperacillin-tazobactam versus carbapenem therapy with and without amikacin as empirical treatment of febrile neutropenia in cancer patients: results of an open randomized trial at a university hospital. Jpn J Clin Oncol. 2010;40(8):761-767.

25. Rolston KV. New trends in patient management: riskbased therapy for febrile patients with neutropenia. Clin Infect Dis. 1999;29(3):515-521.

26. Hess U, Bohme C, Rey K, Senn HJ. Monotherapy with piperacillin/tazobactam versus combination therapy with ceftazidime plus amikacin as an empiric therapy for fever in neutropenic cancer patients. Support Care Cancer. 1998;6(4):402-409.

27. Klastersky J, Ameye L, Maertens J, Georgala A, Muanza F, Aoun M, Ferrant A, et al. Bacteraemia in febrile neutropenic cancer patients. Int J Antimicrob Agents. 2007;30(Suppl 1):S51-59.

28. Sepkowitz KA. Treatment of patients with hematologic neoplasm, fever, and neutropenia. Clin Infect Dis. 2005;40(Suppl 4):S253-256.

29. Gorschluter M, Hahn C, Fixson A, Mey U, Ziske C, Molitor E, Horre R, et al. Piperacillin-tazobactam is more effective than ceftriaxone plus gentamicin in febrile neutropenic patients with hematological malignancies: a randomized comparison. Support Care Cancer. 2003;11(6):362-370.

30. Vural S, Erdem E, Gulec SG, Yildirmak Y, Kebudi R. Imipenem-cilastatin versus piperacillin-tazobactam as monotherapy in febrile neutropenia. Pediatr Int. 2010;52(2):262-267.

31. Viscoli C, Cometta A, Kern WV, Bock R, Paesmans M, Crokaert F, Glauser MP, et al. Piperacillin-tazobactam monotherapy in high-risk febrile and neutropenic cancer patients. Clin Microbiol Infect. 2006;12(3):212-216.

32. Kutluturk F, Uzun S, Tasliyurt T, Sahin S, Barut S, Ozturk B, et al. A Rare Complication of Antibiotic (Piperacillin/ Tazobactam) Therapy: Resistant Hypokalemia. Journal of Medical Cases. 2012;3(6):355-357.

33. Rasool Hassan BA, Yusoff ZB, Othman SB. Fever/clinical signs and association with neutropenia in solid cancer patients--bacterial infection as the main cause. Asian Pac J Cancer Prev. 2010;11(5):1273-1277. 